Research Article

\title{
Effects of Regional Magnetic Field on Rotating MHD Flow Field of Unity Magnetic Prandtl Number
}

\author{
Sheng Lun Hung and Jik Chang Leong \\ Department of Vehicle Engineering, National Pingtung University of Science and Technology, \\ Pingtung 912, Taiwan \\ Correspondence should be addressed to Jik Chang Leong, jcleong@mail.npust.edu.tw
}

Received 29 January 2012; Revised 30 March 2012; Accepted 16 April 2012

Academic Editor: Hiroshi Kanayama

Copyright (C) 2012 S. L. Hung and J. C. Leong. This is an open access article distributed under the Creative Commons Attribution License, which permits unrestricted use, distribution, and reproduction in any medium, provided the original work is properly cited.

\begin{abstract}
This work numerically studies the flow pattern of a magnetic fluid filled within an annulus whose inner cylinder is moving at a constant rotational speed, while the outer cylinder is stationary but under the influence of a nonuniform external magnetic field. The magnetic field consists of four basic configurations, that is, completely circular, semicircular, quarter circular, and alternately quarter circular. The strength of the external magnetic field is characterized using a reference Hartmann number. As the reference Hartmann number increases, the fluid elements need to overcome greater resistance to enter the region with magnetic field. Hence, there always exists an apparent recirculation cell within the region without externally applied magnetic field. The strength and size of the recirculation cell depend on the reference Hartmann number, the number and size of the discrete regions without external magnetic field. Only the shear stress on the moving cylinder always increases in magnitude with the reference Hartmann number and the span of the single external magnetic field region. Splitting and separating the external magnetic field may increase the magnitude of the shear stress on the moving inner cylinder but decrease that on the stationary outer cylinder. If the magnitude of the shear stress on the outer cylinder reduces beyond zero, a shear stress in the opposite sense will increase in magnitude with Hartmann number.
\end{abstract}

\section{Introduction}

The study of magnetohydrodynamics (MHD) has recently become a topic of study which has attracted a lot of attention. In the 1960s, Papell of NASA mixed very fine magnetite particles below $10 \mathrm{~nm}$ with appropriate surfactant so that the nanomagnetite could be effectively dispersed in nonpolar solvents [1]. He then successfully produced a magnetic fluid which had demonstrated many very distinctive physical behaviors. In general, magnetic fluid can 
be classified as either conducting or nonconducting depending on their nature of electric conductivity. Under the influence of an external magnetic field, a conducting magnetic fluid in motion will produce an electromotive force that causes an induced electric current to flow. The presence of this induced electric field then in turn produces an induced magnetic field. At the same time, the induced electric field interacts with the overall magnetic field to produce Lorentz force that acts on the fluid elements. Since the Lorentz force acts to oppose the mechanisms that create it, it therefore generally serves to reduce the magnitude of fluid element velocity field.

As a matter of fact, Hartmann, way ahead of Papell's study, had successfully investigated the nature of magnetic Poiseuille flow based on experimental and theoretical approaches as early as 1937 [2]. His study was later referred to as the well-known Hartmann flow and the dimensionless parameter appeared in the problem was named after him as the Hartmann number. This parameter basically represents the relative importance between the magnetic and the inertial forces. In 1970, Finlayson [3] performed a theoretical study of a magnetic fluid behavior under the influence of perpendicular uniform external magnetic field.

Chang and Lundgren [4] have also performed a rather complete investigation on Hartmann flow. In their paper, they pointed out that the flow at the middle between the upper and lower surfaces tends to become flatter as Ha increases. Not only so, the overall velocity of the fluid decreases with Ha. In 1999, Yamaguchi et al. [5] studied the instability of magnetic fluid in a two-dimensional enclosure subjected to an external magnetic field through experimental and numerical simulations. As of this moment, magnetic fluid is widely employed in various engineering applications.

\section{(a) Medical Therapy}

For cancer detection, magnetic fluids are commonly used as the MRI contrast agents. Moreover, magnetic fluid is also used in experimental cancer treatments named magnetic hyperthermia.

\section{(b) Seals for Electronic Devices}

Magnetic fluids are used to form liquid seals surrounding the driver shafts in hard disk drives. A small amount of oil-based magnetic fluid can be injected into the gap between the magnet and the rotating shaft. The magnetic attraction holds the fluid in place forming a protection that prevents dusts from entering the hard disk drive.

\section{(c) Lubrication}

Oil-based magnetic fluids are also excellent lubricants. They can be injected into the gaps between moving mechanical parts of distinctive speeds. During operation, the leakage of the fluid can be prevented under the influence of magnetism. This feature is highly valued in the industry for it greatly reduces the hassle for consistent lubricant refill [6].

\section{(d) Transportation}

Magnetic fluids are now widely used in the magnetorheological damper, a kind of active suspension system surrounded with an electromagnet. The viscosity of the magnetic fluid in 
this kind of vehicle damper can be dramatically varied to account for the driver preference or the vehicle overall weight. More importantly, its viscosity may be dynamically regulated to improve overall vehicle stability control because the damping factor of the active suspension system can be adjusted once every millisecond in response to actual road conditions. As a matter of fact, magnetorheological dampers are also used in helicopter cockpit seats as safety devices which serve to significantly decrease the rate of permanent injury during a crash.

\section{(e) Energy Conversion}

The energy conversion device using magnetic fluid is attractive for space use or in a situation where no maintenance is required. Yamaguchi et al. [7] have designed and constructed a direct-heat-to-power energy conversion device that makes use of temperature-sensitive magnetic fluid.

For a very long period of time, fluid behaviors between a pair of independently rotating cylinders have been investigated extensively. Perhaps all studies in rotational MHD flow trace back to the work by Taylor $[8,9]$ who has investigated both theoretically and experimentally the stability of the classical Couette-Taylor flow. Shortly after Taylor's discovery of the onset of axisymmetric Taylor vortices, Jeffreys [10] has extended the concept of Couette-Taylor flow and shown that the earth rotational motion has a negligible influence on the mantle convective motion. On the other hand, Meksyn [11] has investigated a similar problem using a different asymptotic method of integration. Later, Coles and van Atta [1214] disclose nonaxisymmetric spiral vortices as a result of counter-rotating cylinders. Since then, just to name a few, many monumental studies [15-27] mostly deal with flow instability have been reported laying a solid foundation and inspiration for numerous future research topics and activities.

As a matter fact, Chandrasekhar [27] has recognized the need for further understanding of magnetic fluid instability associated to rotating cylinders. He extended the work by Taylor, Jeffreys, and Meksyn and considered an electrically conducting under the influence of an axial magnetic field. There are several factors affecting the effectiveness of the magnetic field in suppressing the onset of thermal instability. However, instability suppression due to the presence of the magnetic field is always more pronounced for rotational motion than for differential heating from below. While there were many research works related to a disk configuration, Donnelly et al. [28-30], Brahme [31], Ji et al. [32, 33] have contributed to the understanding of megnetorotational instability (MRI) in rotating fluid.

Despite the vast collection of MHD Couette flow problems, rarely found are those not tackling instability problems. Willis and Barenghi [34] investigated the response of a conducting liquid in a three dimensional cylindrical geometry subjected to an imposed axial magnetic field. The patterns of a typical nonlinear steady hydromagnetic flow were shown. Because of the magnetic field, the Taylor cells found in classical Coutte-Taylor flow are apparently elongated in the axial direction.

Szklarski and Rüdiger [35, 36] have actually simulated a magnetorotational MHD Taylor-Couette flow with an external helical magnetic field at small Prandtl number limits. Through plots of stream function contour, they showed the existence of drifting vortex pairs within an infinitely long annular space. For an enclosure with a motionless bottom endplate and a rotating upper endplate, a traveling wave propagates in the upward axial direction dramatically distorting the drifting vortex pairs observed in infinitely long annulus. The formation of Ekman-Hartmann layer is observed near the endplates where a Hartmann current exists and penetrates the bulk of the conducting fluid. 
The purpose of this paper is to numerically study the flow pattern of a magnetic fluid filled within an annulus under the influence of different nonuniform externally applied magnetic field configurations. Based on the flow field obtained for different magnetic field configurations and field strengths, the shear stress along the moving cylinder is evaluated.

The description for the formulation of the magnetic fluid confined within a pair of infinitely long cylinders is given in Section 2. The governing equations along with the appropriate boundary conditions were made dimensionless and numerical approach was employed to solve the equations. The flow patterns of the magnetic fluid under the influence of the nonuniform externally applied magnetic field are investigated in Section 3. It is shown that the flow pattern depend strongly on the Hartmann number, that is, the strength of the external magnetic field. In particular, it is shown that the configuration of the external magnetic field plays a very important role in local acceleration and deceleration of the magnetic fluid. The change in shear stress due to the change in flow pattern is also documented. Finally, some important findings are reported in Section 4.

\section{Governing Equations}

Current work numerically simulates the flow field of a two-dimensional cylindrical magnetic Couette flow. The polar coordinate system is represented by $(r, \theta)$, while the velocity and external magnetic fields are represented by $\left(u_{r}, u_{\theta}\right)$ and $\left(B_{r}, B_{\theta}\right)$, respectively. The radii of the inner and outer cylinders are represented by $r_{i}$ and $r_{0}$. Also, the inner cylinder rotates at a constant speed $\Omega$.

The behaviors of electromagnetism are governed by the Maxwell's equations. As a matter of fact, these equations are a collection of four fundamental governing equations individually known as the Gauss's law, the Gauss's law for magnetism, the Faraday's law of induction, and the Ampère's law with Maxwell's correction. Together with the so-called material equations, these equations are mathematically given as [37]

$$
\begin{gathered}
\nabla \cdot \vec{B}=0, \\
\nabla \times \vec{E}=-\frac{\partial \vec{B}}{\partial t}, \\
\nabla \times \vec{H}=\vec{j}, \\
\nabla \cdot \vec{D}=0,
\end{gathered}
$$

where the above parameters are interrelated through the following relationships:

$$
\begin{gathered}
\vec{H}=\frac{1}{\mu} \vec{B}, \\
\vec{j}=\sigma(\vec{E}+\vec{V} \times \vec{B}), \\
\vec{D}=\varepsilon \vec{E} .
\end{gathered}
$$


Combining (2.1), (2.2), (2.3), (2.5), and (2.6), the general magnetic field equation for any magnetic fluid can be obtained in the following form:

$$
\frac{\partial \vec{B}}{\partial t}+(\vec{V} \cdot \nabla) \vec{B}+(\nabla \cdot \vec{V}) \vec{B}=(\vec{B} \cdot \nabla) \vec{V}+\frac{1}{\mu \sigma} \nabla^{2} \vec{B}
$$

Although one can clearly understand the relationships and behaviors between the magnetic and electric fields, the theory of electromagnetism has also suggested that an electromagnetic force namely the Lorentz force will be produced in the magnetic fluid as it flows in the region under the influence of magnetic field. This force later tends to influence the motion of the fluid elements. For this reason, the magnetic, electric, and flow fields are interinfluential. The conservations of mass and momentum of the fluid are governed by the continuity and Navier-Stokes equation as

$$
\begin{gathered}
\frac{D \rho}{D t}=0 \\
\frac{D(\rho \vec{V})}{D t}=-\nabla p+\rho \vec{g}+\frac{1}{\mu}(\nabla \times \vec{B}) \times \vec{B}+\mu \nabla^{2} \vec{V} .
\end{gathered}
$$

Current study assumes that the magnetic fluid is steady, incompressible, and laminar while the gravitational acceleration is negligible. Equation (2.9) simplifies as

$$
\begin{gathered}
\nabla \cdot \vec{V}=0, \\
(\vec{V} \cdot \nabla) \vec{V}=-\frac{1}{\rho} \nabla p+\vec{g}+\frac{1}{\mu \rho}(\nabla \times \vec{B}) \times \vec{B}+v \nabla^{2} \vec{V} .
\end{gathered}
$$

With the help of continuity equation, (2.8) can be further simplified to give

$$
(\vec{V} \cdot \nabla) \vec{B}=(\vec{B} \cdot \nabla) \vec{V}+\frac{1}{\mu \sigma} \nabla^{2} \vec{B}
$$

The external magnetic field varies as a function of radial direction, that is, $B_{0} / r$, where $B_{0}$ will then be used to define the reference Hartmann number in current study. The dynamical state of the magnetic fluid can therefore be determined by solving (2.10)-(2.12) simultaneously. This set of equations is apparently too complicated to be solved directly. To further simplify it, stream function $\psi$ is introduced to eliminate the continuity equation and thus to reduce the number of equation needed to solve simultaneously. The stream function $\psi$ is defined such that

$$
u_{r}=\frac{1}{r} \frac{\partial \psi}{\partial \theta}, \quad u_{\theta}=-\frac{\partial \psi}{\partial r}
$$


where $u_{r}$ and $u_{\theta}$ are the dimensionless radial and azimuthal components of velocity. On the other hand, the following dimensionless parameters are also introduced to normalize the remaining set of equations:

$$
\begin{array}{cc}
r^{*}=\frac{r}{r_{i}}, \quad \psi^{*}=\frac{\Psi}{v}, \quad \Omega^{*}=\frac{\Omega}{v / r_{i}^{2}}, \\
\omega^{*}=\frac{\omega}{v / r_{i}^{2}}, \quad A^{*}=\frac{A}{B_{0}}, \quad \text { На }=\frac{B_{0} a}{r_{i}} \sqrt{\frac{\sigma}{\rho v}} .
\end{array}
$$

The parameter Ha is referred to as the reference Hartmann number because the term $B_{0} / r_{i}$ is actually the amount of reference external magnetic field density at $r_{i}$. Clearly, a larger Ha indicates a stronger external magnetic field $B_{0}$. The parameters appeared within the square root are merely electrical and mechanical properties of the magnetic fluid. To simplify the formulation, the distribution of the magnetic potential $A$ is more frequently solved to replace the magnetic field density. The magnetic potential $A$ is defined similar to the stream function and is given as below for a two-dimensional formulation

$$
B_{r}=\frac{1}{r} \frac{\partial A}{\partial \theta}, \quad B_{\theta}=-\frac{\partial A}{\partial r} .
$$

Neglecting displacement currents, induced magnetic field, dissipation, and Joule heating, the Navier-Stokes equations can be transformed into the following nondimensional stream function-vorticity form after dropping the asterisk in (2.14) for simplicity:

$$
\begin{aligned}
& \frac{1}{r} \frac{\partial}{\partial r}\left(\omega \frac{\partial \psi}{\partial \theta}\right)-\frac{1}{r} \frac{\partial}{\partial \theta}\left(\omega \frac{\partial \psi}{\partial r}\right) \\
& =\frac{1}{r} \frac{\partial}{\partial r}\left(r \frac{\partial \omega}{\partial r}\right)+\frac{1}{r^{2}} \frac{\partial^{2} \omega}{\partial \theta^{2}}-\frac{\mathrm{Ha}^{2}}{r^{2}}\left(\frac{\partial A}{\partial \theta} \frac{\partial}{\partial r}-\frac{\partial A}{\partial r} \frac{\partial}{\partial \theta}\right)\left[\frac{\partial}{\partial r}\left(r \frac{\partial A}{\partial r}\right)+\frac{1}{r} \frac{\partial^{2} A}{\partial \theta^{2}}\right]
\end{aligned}
$$

where the definition of dimensionless vorticity is given below without asterisk

$$
\frac{1}{r} \frac{\partial}{\partial r}\left(r \frac{\partial \psi}{\partial r}\right)+\frac{1}{r^{2}} \frac{\partial^{2} \psi}{\partial \theta^{2}}=-\omega
$$

To be consistent, the dimensional magnetic field equation, (2.12), was also nondimensionalized and simplified after some manipulation to yield

$$
\begin{aligned}
& \frac{1}{r} \frac{\partial}{\partial r}\left(A \frac{\partial \psi}{\partial \theta}\right)-\frac{1}{r} \frac{\partial}{\partial \theta}\left(A \frac{\partial \psi}{\partial r}\right) \\
& =\frac{1}{r} \frac{\partial}{\partial r}\left(\omega \frac{\partial A}{\partial \theta}\right)-\frac{1}{r} \frac{\partial}{\partial \theta}\left(\omega \frac{\partial A}{\partial r}\right)+\frac{1}{P_{m}}\left[\frac{1}{r} \frac{\partial}{\partial r}\left(r \frac{\partial A}{\partial r}\right)+\frac{1}{r^{2}} \frac{\partial^{2} A}{\partial \theta^{2}}\right],
\end{aligned}
$$

where $P_{m}$ is the magnetic Prandtl number defined as $P_{m}=\mu \sigma v$. 


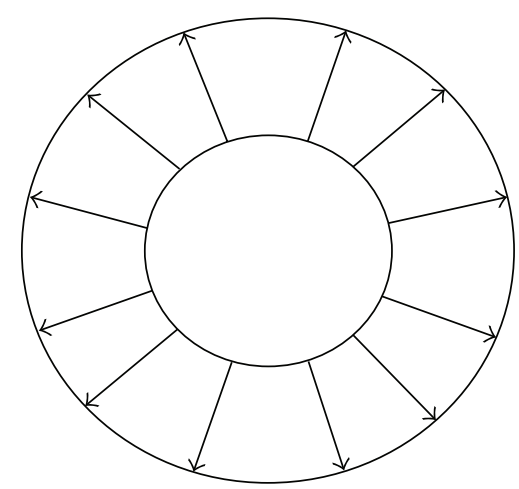

(a)

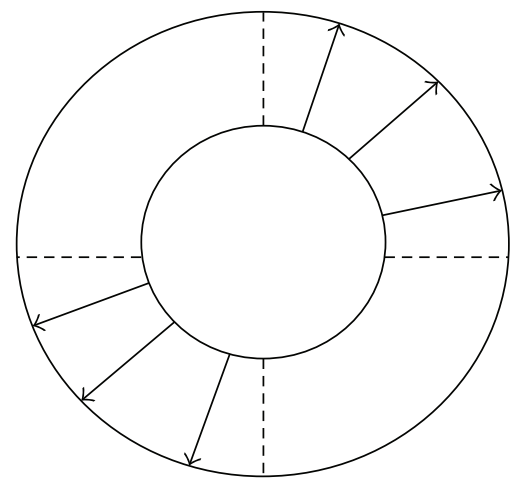

(c)

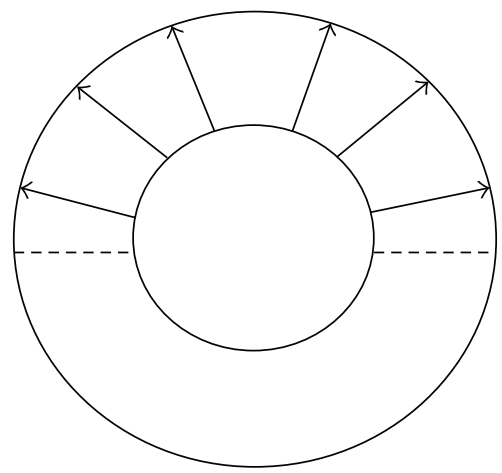

(b)

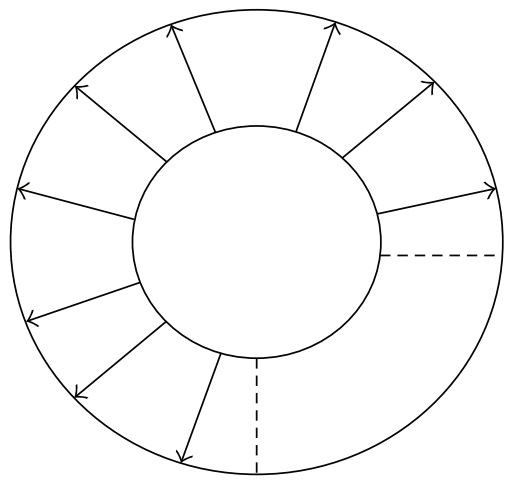

(d)

Figure 1: Distribution of externally applied magnetic fields: (a) uniform, (b) a semicircular, (c) two-quarter circular, and (d) a three-quarter circular.

Appropriate boundary conditions for the velocity field are imposed by specifying the values of dimensionless stream function on the surface of the inner and outer cylinders. After dropping the asterisk, they are given in terms of dimensionless rotational speed of the inner cylinder as

Inner cylinder,

$$
\psi=\frac{r_{o}-r_{i}}{r_{i}} \Omega \text { at } r=1 \text {, }
$$

Outer cylinder,

$$
\psi=0 \quad \text { at } r=\frac{r_{O}}{r_{i}} .
$$

Other than the above velocity boundary conditions, an external magnetic field must be imposed on the magnetic fluid. The various configurations of the externally applied magnetic field considered in this study are shown in Figure 1. The dimensionless magnetic 


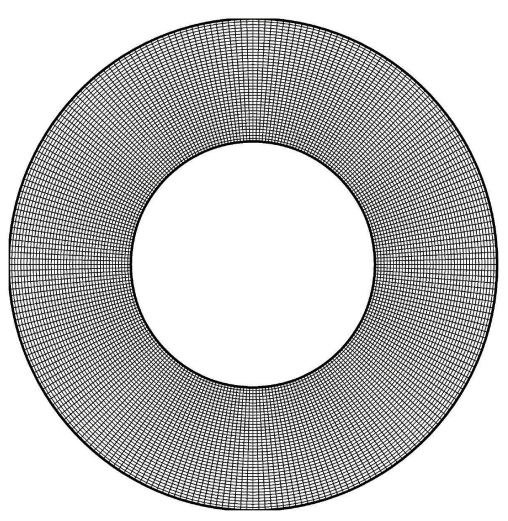

Figure 2: Grid distribution.

potential within the region $\mathcal{R}$ including its inner and outer bounds where the magnetic field is externally applied is given as

Region with magnetic field,

$$
A=\theta \quad \text { for } 1 \leq r \leq \frac{r_{O}}{r_{i}}, \theta \subset \mathcal{R},
$$

Region without magnetic field,

$$
A=0 \quad \text { for } 1 \leq r \leq \frac{r_{o}}{r_{i}}, \theta \not \subset R \text {. }
$$

In the present work, a finite difference method was employed. The governing equations (2.16)-(2.18) were approximated by algebraic equations at the nodal points over the computational domain. A finite volume was constructed surrounding each nodal point. The governing differential equations were then integrated over the control volume. This ensures that the conservation laws were satisfied both over the control volume as well as the computational domain. This numerical method has been successfully employed by the authors [38]. The geometry and grid system of the annular space was constructed using a structured orthogonal mesh system. The grid distribution in this work was $40 \times 260$, as shown in Figure 2. Under- and over-relaxation was employed for most of the calculations to ensure the efficiency and accuracy of the numerical results. The reference Hartmann number investigated in this work includes 1, 3, 5, 10, 20, 30, 40, and 50. A grid refinement test had been performed and it was found that there was no significant improvement on present computational results even if the number of grid was increased up to 8 times denser than the current one.

\section{Results and Discussion}

Since the magnetic fluid flow in the annular space is subjected to an external magnetic field, its fluid elements experience either a local acceleration or a local deceleration. It has long 


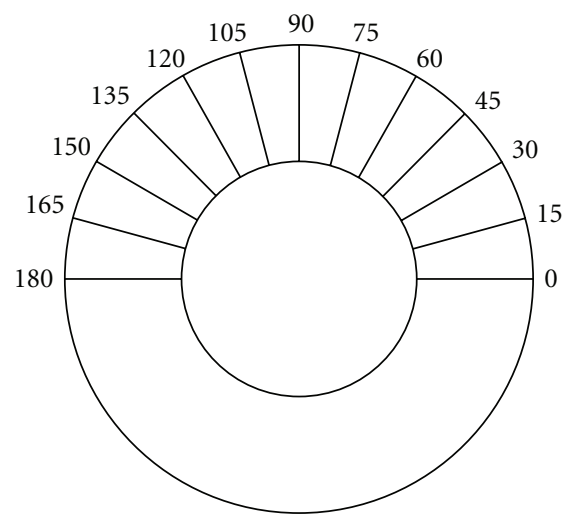

Figure 3: Locations where the velocity profiles are displayed.

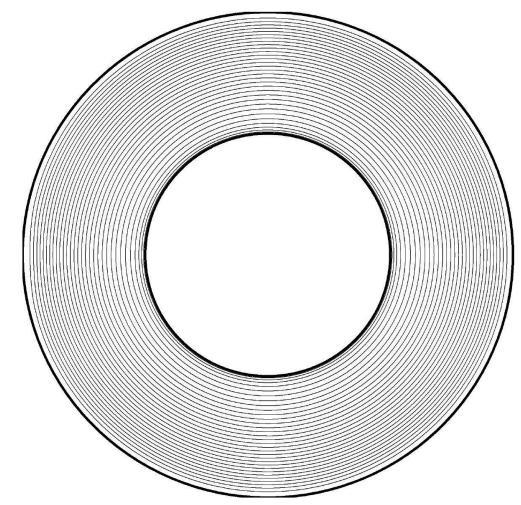

Figure 4: Distribution of the contours of stream function for a uniform external magnetic fluid of Ha = $30(\Delta \psi=0.0025)$.

been recognized through various studies that as Ha increases, the velocity close to the inner cylinder decelerates while that close to the outer cylinder accelerates. Not only so, the velocity gradient at the surfaces of both the inner and outer cylinders increases with Ha. In current study in which the external magnetic field is regional, it is found that the velocity gradient becomes relatively more obvious when $\mathrm{Ha}>20$. For this reason, the discussion that follows will only focus on the scenario where $\mathrm{Ha}=30$ and 50 . In order to further examine these phenomena, this paper divides the upper half section of annulus (i.e., $0^{\circ} \leq \theta \leq 180^{\circ}$ ) into 12 sections which are $15^{\circ}$ wide. These cross-sections between these sections are shown in Figure 3.

The contour of stream functions for $\mathrm{Ha}=30$ is presented in Figure 4. Since the external magnetic field is completely circular within the annular space, the flow patterns are certainly axis-symmetrical. This fact is evident from the distribution of stream function contours. It can be seen that the contours of stream functions appear to be a family of concentric circles. In other words, the magnetic fluid is merely a swirl flow under the influence of the complete circular external magnetic field proving the fact that the flow field is completely one-dimensional and angular independent. 


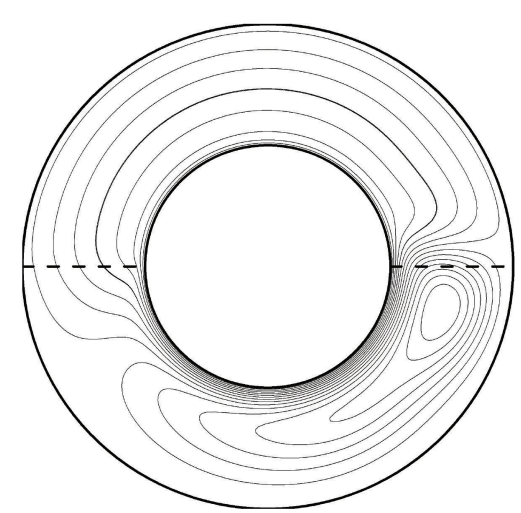

Figure 5: Distribution of the contours of stream function for a semicircular external magnetic fluid of $\mathrm{Ha}=30(\Delta \psi=0.0025)$.

Figure 5 shows the contours of stream functions for the magnetic fluid subjected to a semicircular external magnetic field of $\mathrm{Ha}=30$. Unlike the previous case, there exists in the lower portion of the annular space an additional recirculation cell rotating in the clockwise direction. The eye of the cell is located to the right side of the annulus in the region without external magnetic field. Clearly, the flow pattern is no longer axis-symmetrical. Remember that the magnetic field is only applied in the upper half of the annulus. In this region with an external magnetic field, the magnetic fluid simply flows in the counterclockwise direction. In the lower half where the external magnetic field is absent, the region is mostly occupied by a recirculation cell. The formation of the recirculation cell is solely due to the presence of the external magnetic field in the upper half region. In the upper half region, the fluid elements generally slow down under the influence of the Lorentz force. Prior to entering the upper half region, the fluid elements in the lower half region has experienced a considerable flow resistance. Hence, some of the fluid elements are forced to flow radially outward similar to the phenomena discovered for flow impingement. This radially outward flow eventually forms the recirculation cell. It is remarkable to see that only a very thin layer of magnetic fluid right next to the inner rotating cylinder is capable of penetrating the upper half region because the flow momentum at this region is continuously supplied by the rotation of the inner cylinder. Once it enters the upper region where the recirculation cell is no longer present, it expands almost radially and therefore fills the entire gap between the inner and outer cylinders. As it leaves the upper half region and returns to the lower half region, its fluid elements are squeezed radially inwards by the recirculation cell. Even though the phenomena of magnetic fluid entering and leaving the upper half region is somewhat similar (but in a reversed fashion), the change in velocity field is more abrupt for the magnetic fluid elements entering than leaving the region subjected to an external magnetic field.

In Figure 6, $R^{*}=0$ and $R^{*}=1$ indicate the locations of the inner and outer cylinders, respectively. Here, the aforementioned radial coordinate was scaled through $R^{*}=\left(r-r_{i}\right) /\left(r_{o}-\right.$ $\left.r_{i}\right)$. Similarly, the angular velocity component $u_{\theta}$ of the magnetic fluid was calculated through (2.10) and then normalized by the linear velocity of the inner cylinder, that is, $V^{*}=u_{\theta} / \omega r_{i}$. Apparently, the fluid elements on the inner cylinder move at a finite tangential velocity while those on the outer cylinder remain motionless. Furthermore, there exists a section close to the outer cylinder whose angular velocity component is less than zero at $\theta=0^{\circ}$ indicating that the fluid elements at this section actually flow in the direction opposite to the direction 


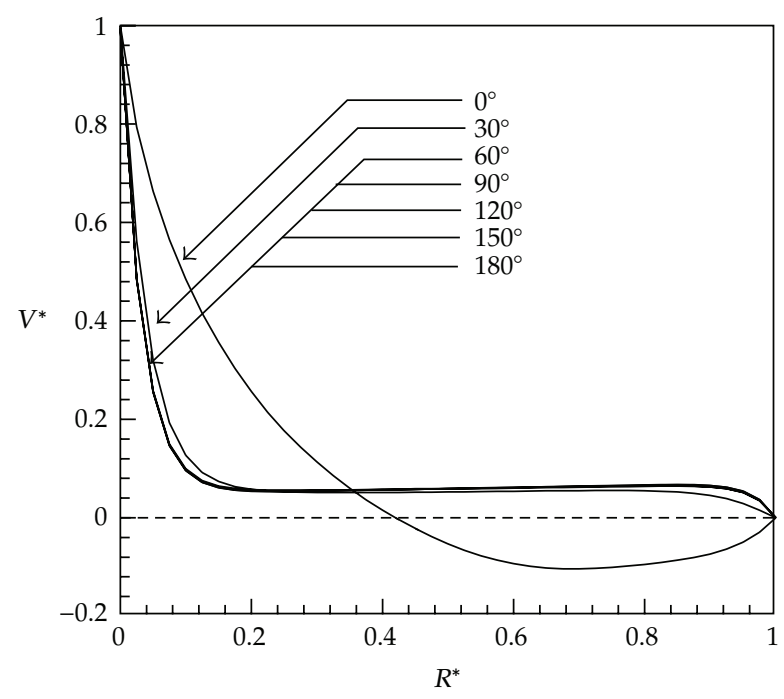

Figure 6: Angular velocity profiles at $0^{\circ} \leq \theta \leq 180^{\circ}$ subjected to a semicircular external magnetic field.

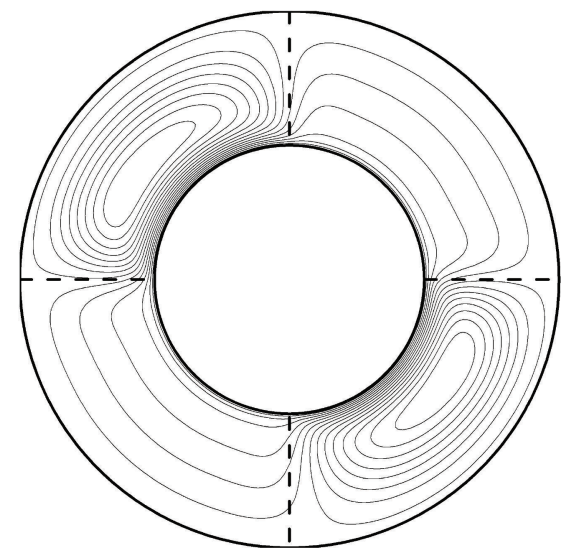

Figure 7: Distribution of the contours of stream function for two-quarter circular external magnetic fluids of $\mathrm{Ha}=30(\Delta \psi=0.00025)$.

of the rotating inner cylinder. At other locations, that is, $30^{\circ} \leq \theta \leq 180^{\circ}$, the angular velocity profiles are almost identical. Based on this figure, it is not difficult to see that the magnitude of the normalized angular velocity $V^{*}$ in the region corresponding to $0.12 \leq R^{*} \leq 0.84$ and $30^{\circ} \leq \theta \leq 180^{\circ}$ is about 0.06 . This implies that the rotational speed in this region is inversely proportional to its radial distance.

If the external magnetic field is applied at the first and third quadrants, the flow patterns can be expected to bear some resemblances with that for a semicircular magnetic field. Since there are two discrete regions without external magnetic field in this case, there surely exist two recirculation cells within these regions, as clearly displayed in Figure 7. However, the strength of these two cells is actually weaker than the single cell for the case shown in Figure 5. This can be imagined as if the single recirculation cell in Figure 5 is forced to split into two recirculation cells by the presence of the additional region with external 


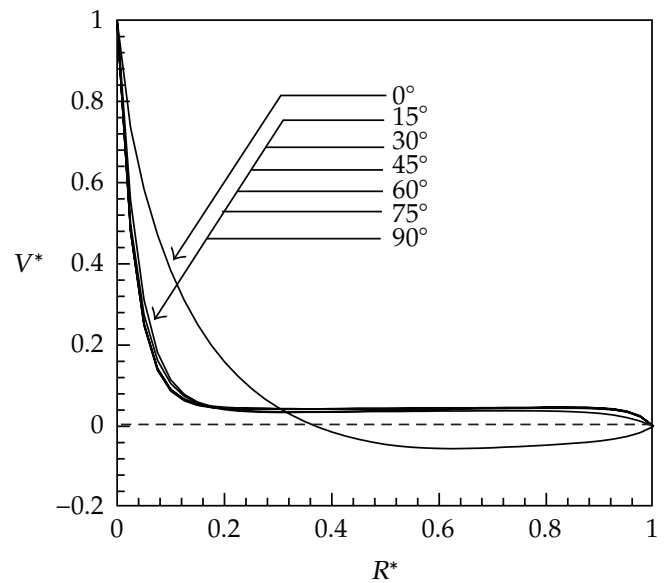

(a)

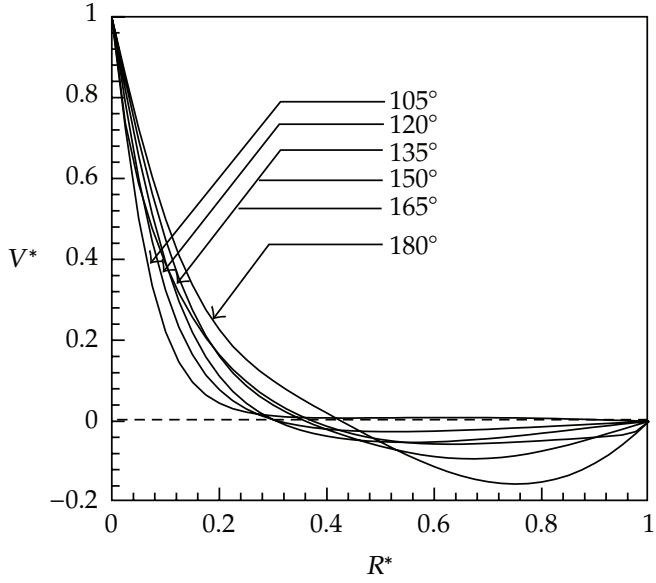

(b)

Figure 8: Angular velocity profiles subjected to two-quarter circular external magnetic fields at (a) $0^{\circ} \leq \theta \leq$ $90^{\circ}$ and (b) $105^{\circ} \leq \theta \leq 180^{\circ}$.

magnetic field. As the single larger recirculation cell splits into two smaller cells, their strength apparently weakens. This means the fluid elements in Figure 7 circulate at a lower velocity. In the regions with external magnetic fields, the distribution of stream function contours in general is less dense in Figure 7 in comparison with those depicted in Figure 5. This clearly suggests that, except in the vicinity very close to the rotating inner cylinder, the majority fluid elements flow slower if the single region with an external magnetic field is split and separated. This phenomenon implies the decrease in flow kinetic energy in the annular space is attributed to the effect of additional flow deceleration and acceleration prior to crossing the interface between the regions with and without external magnetic field. Based on a careful comparison, it was also found that the flow in this case has to squeeze itself through a slightly narrower gap between the recirculation cell and the surface of the inner rotating cylinder.

To further understand the nature of the magnetic fluid flow, the velocity profiles in the upper half region of the annulus are plotted in Figure 8. It is observed that at $\theta=$ $0^{\circ}$, there is a large section where flow reversal takes place. Among the various velocity profiles demonstrated, the angular velocity component is all greater than 0 for $15^{\circ} \leq \theta \leq$ $120^{\circ}$. Although the external magnetic field is only applied at $0^{\circ} \leq \theta \leq 90^{\circ}$, the upstream recirculation cell actually stretches beyond the interface at $\theta=0^{\circ}$ while the counter-clockwise swirl flow extends over $\theta=105^{\circ}$ which is beyond the other interface at $\theta=90^{\circ}$. In this region, the velocity profiles at $30^{\circ} \leq \theta \leq 90^{\circ}$ are almost the same.

The flow pattern of the magnetic fluid in the annular space under the influence of a three-quarter-circular external magnetic field is shown in Figure 9. In this scenario, only the forth quadrant is not subjected to an external magnetic field. In Figure 9(a), the strength of the external magnetic field is associated to $\mathrm{Ha}=30$. The flow patterns of fluid elements entering the region subjected to an external magnetic field are very similar to the previous cases. However, the flow pattern of the fluid elements leaving the region is very distinctive. Under the influence of the external magnetic field spanning over the upper half region as in Figure 5, the fluid elements are enormously squeezed so that they manage to complete a cycle. If the external magnetic field is split, separated, and applied alternatively over the upper and lower half semiannular spaces as in Figure 7, the fluid elements still need to force 


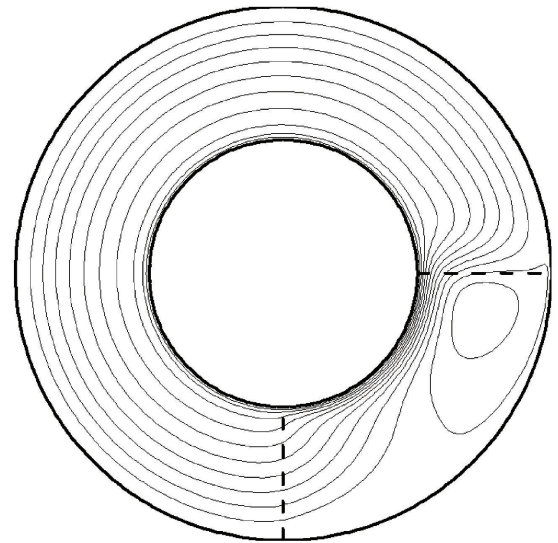

(a)

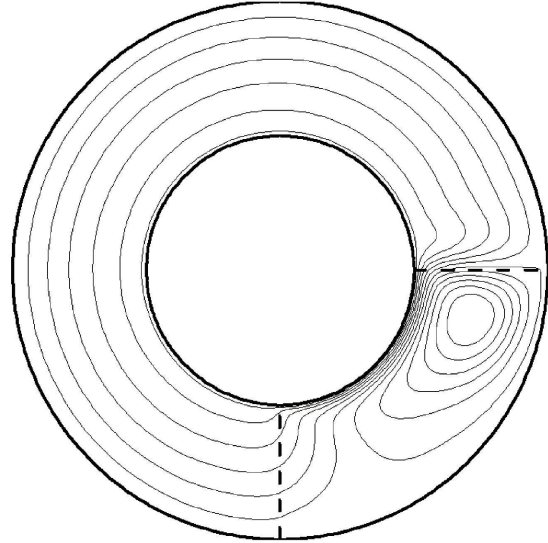

(b)

Figure 9: Distributions of the contours of stream function for a three-quarter-circular external magnetic fluids of (a) $\mathrm{Ha}=30$ and (b) $\mathrm{Ha}=50(\Delta \psi=0.0025)$.

their way through the annular space. When an additional external magnetic field is applied at the second quadrant as in Figure 9(a), the fluid elements actually experience less resistance when flowing through the region without external magnetic field.

Based upon these figures, it is remarkable to find that the strength and size of the recirculation cell are strongly dependent on the number of the discrete regions without external magnetic field and their span in the angular direction. Among these three external magnetic field configurations, the recirculation cell is the weakest when only a quarter of the annular space is free of external magnetic field. As the span of this region without external magnetic field is doubled, the strength of the cell increases due to the decrease in Lorentz force, as evidently shown in Figure 5. If the second and third quadrants of a semicircular external magnetic field (in Figure 5) are interchanged to form the two quartercircular external magnetic fields, the recirculation cell is also split into two individual cells whose strengths are apparently less than the previous one.

Figure 9 (b) shows the flow pattern for the same conditions except that Ha is increased to 50. The flow pattern is quite similar to that in Figure 9(a) except at the regions in the vicinity of the recirculation cell. Because of the increase in the reference Hartmann number, the corresponding recirculation cell grows in strength and as a result it leaves the swirl flow less space to go around. Also for this reason, the change in flow direction prior to the formation of the recirculation cell is much more dramatic for $\mathrm{Ha}=50$ than for $\mathrm{Ha}=30$. This also clearly proves that the strength of the recirculation cell for $\mathrm{Ha}=50$ is greater between the two cases. As the fluid elements enter the region with an external magnetic field of $\mathrm{Ha}=50$, they also tend to change their flow directions more rapidly under the influence of stronger Lorentz force.

If the external magnetic field conditions shown in Figure 9 are interchanged to form a quarter circular magnetic field, the general flow patterns interchange between the regions with and without external magnetic field. As shown in Figure 10, the strength of the recirculation cell actually increases tremendously as evidently depicted by the denser distribution of the contours surrounding the eye of the recirculation cell. More surprisingly, 


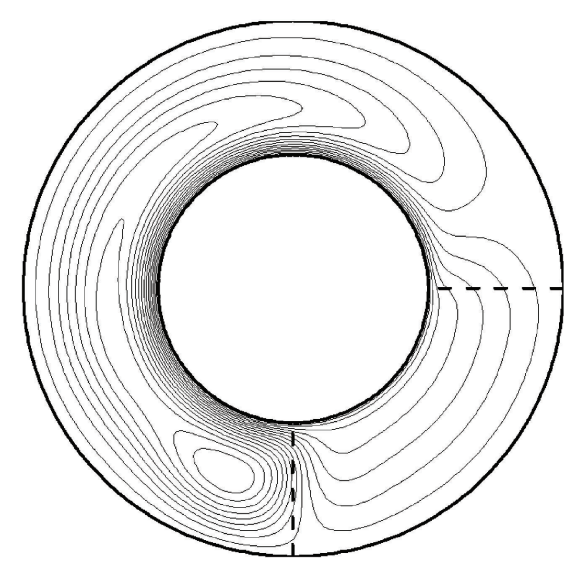

Figure 10: Distributions of the contours of stream function for a quarter-circular external magnetic fluids of $\mathrm{Ha}=30(\Delta \psi=0.0025)$.

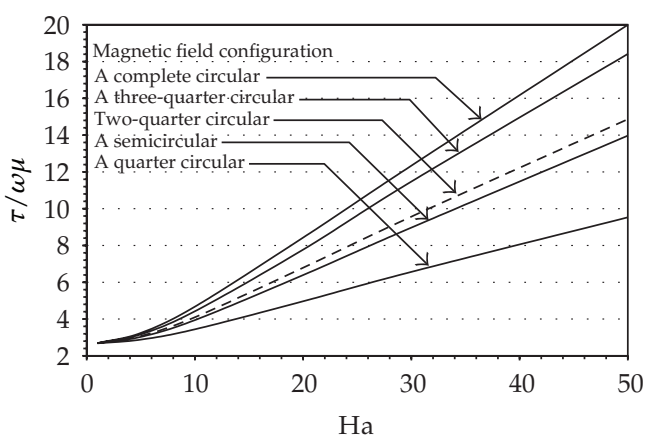

(a)

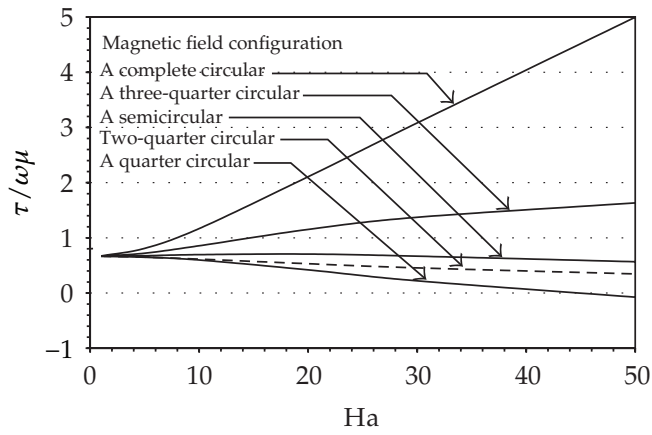

(b)

Figure 11: Variation of dimensionless shear stress on cylindrical surface: (a) inner and (b) outer.

this recirculation cell is even stronger than the one shown in Figure 9(b), which corresponds to $\mathrm{Ha}=50$.

The shear stress $\tau$ on the inner and outer cylinders were nondimensionalized by the product of the inner cylinder rotational speed $\omega$ and the fluid dynamic viscosity $\mu$. The dependence of these dimensionless shear stresses on the external magnetic field configuration and Ha are displayed in Figure 11. As Ha increases, the shear stress on the inner cylinder always increases but that on the outer cylinder does not necessarily follows this rule. In fact, the formation of the recirculation cell in the region without external magnetic field serves to reverse the shear stress the outer cylinder surface experiences. For this reason, it is found there exists a critical reference Hartmann number beyond which the shear stress on the outer cylinder acts on the opposite direction if only an external magnetic field is applied to a quarter of the annular space. Not only so, whenever the external magnetic field covers less than half of the annular space, the magnitude of the shear stress on the outer cylinder will tends to decreases and will eventually replaced by a growing shear stress in the opposite sense if the Hartman number is large enough. Also in these figures, the effect of splitting and separating the external magnetic field on the shear stress on the inner and outer cylinders is investigated. The shear stress associated with the semicircular magnetic field is found to 
increase in magnitude on the inner cylinder but to decrease in magnitude on the outer one as it is split to form two quarter-circular magnetic field (represented by the broken lines). As the Hartmann number is increases, this magnitude continues to reduce to zero. Then, a shear stress in the opposite sense is anticipated to grow in magnitude. Again, this is strictly related to the nature of the recirculation cells. As the magnetic field is split, the recirculation cell corresponding to the semicircular magnetic field is also split to form two weaker recirculation cells. Because of the presence of these two recirculation cells, the shear flow next to the inner cylinder is more difficult to squeeze through the annular space leading to an increase in shear stress magnitude. On the other hand, the two weaker recirculation cells exert greater reversed friction force and therefore reduce the magnitude of the shear stress on the outer cylinder.

\section{Summary}

A computational study of magnetic fluid in an annular space subjected to a rotating inner cylinder and nonuniform external magnetic fields is accomplished in this work. Based on current investigation, the following conclusions can be drawn.

(1) The presence and absence of the external magnetic field brake the fluid elements prior to entering the region with external magnetic field. This leads to the generation of a recirculation cell.

(2) In the annular space, a recirculation cell always occupies every discrete region without external magnetic field.

(3) The strength and size of the recirculation cell depend on the strength of the external magnetic field, the number of discrete regions without external magnetic field, and their span.

(4) The strength of the recirculation cell is inversely proportional to the span in the angular direction of the region subjected to external magnetic field.

(5) The shear stress on the moving inner cylinder increases with the reference Hartmann number and the span of the single external magnetic field region.

(6) The magnitude of the shear stress on the stationary outer cylinder may increase or decrease with the reference Hartmann number depending on the external magnetic field configuration.

(7) Splitting and separating an external magnetic field may cause the magnitude of the shear stress on the moving inner cylinder to increase but that on the stationary outer cylinder to decrease.

\section{Nomenclature}

A : Magnetic scalar potential, A

a: Gap width between cylinders, $\mathrm{m}$

$B$ : Magnetic field density, $\mathrm{T}$

$B_{0}$ : External magnetic field density, $\mathrm{T}$

$D$ : Electric displacement field, $\mathrm{C} / \mathrm{m}^{2}$

E: Electric field, $\mathrm{V} / \mathrm{m}$ 
$H$ : Magnetized field, $\mathrm{A} / \mathrm{m}$

Ha: Hartmann number,-

$j$ : Current density, A $/ \mathrm{m}^{2}$

$P_{m}$ : Magnetic Prandtl number,-

$q$ : Electric charge, $\mathrm{C}$

$r$ : Radial coordinate, $\mathrm{m}$

$t$ : Time, $\mathrm{s}$

$u_{r}:$ Radial velocity, $\mathrm{m} / \mathrm{s}$

$u_{\theta}:$ Angular velocity, $\mathrm{m} / \mathrm{s}$.

\section{Greek Symbols}

$\varepsilon$ : Permittivity, $\mathrm{F} / \mathrm{m}$

$v$ : Fluid kinematic viscosity, $\mathrm{m}^{2} / \mathrm{s}$

$\mu$ : Fluid dynamic viscosity, $\mathrm{kg} / \mathrm{m} \cdot \mathrm{s}$

$\theta$ : Angular coordinate,-

$\rho$ : Fluid density, $\mathrm{kg} / \mathrm{m}^{3}$

$\sigma$ : Electric conductivity, $\Omega^{-1} \mathrm{~m}^{-1}$

$\omega$ : Rotational speed of the inner cylinder, $\mathrm{rad} / \mathrm{s}$.

\section{References}

[1] S. S. Papell, Low viscosity magnetic fluid obtained by the colloidal suspension of magnetic particles, U.S. Patent No. 3215527, 1965.

[2] J. Hartmann, "Hg dynamics I. Theory of the laminar flow of an electrically conductive liquid in a homogeneous magnetic," FieldMathematisk-Fysiske Meddelelser, vol. 15, no. 6, pp. 1-27, 1937.

[3] B. A. Finlayson, "Convective instability of ferromagnetic fluids," Journal of Fluid Mechanics, vol. 40, no. 4, pp. 753-767, 1970.

[4] C. C. Chang and T. S. Lundgren, "Duct flow in magnetohydrodynamics," Zeitschrift Fuangezwandte Mathematik and Physik, vol. 12, pp. 100-114, 1961.

[5] H. Yamaguchi, I. Kobori, and Y. Uehata, "Heat transfer in natural convection of magnetic fluids," Journal of Thermophysics and Heat Transfer, vol. 13, no. 4, pp. 501-507, 1999.

[6] R. E. Rosensweig, "An introduction to ferrohydrodynamics," Chemical Engineering Communications, vol. 67, no. 1, pp. 1-18, 1988.

[7] H. Yamaguchi, A. Sumiji, S. Shuchi, and T. Yonemura, "Characteristics of thermo-magnetic driven motor using magnetic fluid," Journal of Magnetism and Magnetic Materials, vol. 272-276, no. 3, pp. 2362-2364, 2004.

[8] G. I. Taylor, "Stability of a viscous liquid contained between two rotating cylinders," Philosophical Transactions of the Royal Society A, vol. 223, pp. 289-343, 1923.

[9] G. I. Taylor, "Fluid friction between rotating cylinders," Proceedings of the Royal Society A, vol. 157, pp. 546-564, 1936.

[10] H. Jeffreys, "Some cases of instability in fluid motion," Proceedings of the Royal Society A, vol. 118, pp. 195-208, 1928.

[11] D. Meksyn, "Stability of viscous flow between rotating cylinders. I," Proceedings of the Royal Society. London. Series A, vol. 187, pp. 115-128, 1946.

[12] D. Coles, "Transition in circular Couette flow," Journal of Fluid Mechanics, vol. 21, pp. 385-425, 1965.

[13] D. Coles and C. van Atta, "Measured distortion of a laminar circular Couette flow by end effects," Journal of Fluid Mechanics, vol. 25, pp. 513-521, 1966.

[14] C. van Atta, "Exploratory measurements in spiral turbulence," Journal of Fluid Mechanics, vol. 25, pp. 495-512, 1966.

[15] E. R. Krueger, A. Gross, and R. C. DiPrima, "On the relative importance of Taylor-vortex and nonaxisymmetric modes in flow between rotating cylinders," Journal of Fluid Mechanics, vol. 24, pp. 521538, 1966. 
[16] H. A. Snyder, "Stability of rotating Couette flow. I. Asymmetric waveforms," Physics of Fluids, vol. 11, no. 4, pp. 728-734, 1968.

[17] H. A. Snyder, "Stability of rotating Couette flow. II. Comparison with numerical results," Physics of Fluids, vol. 11, no. 8, pp. 1599-1605, 1968.

[18] H. A. Snyder, "Waveforms in rotating Couette flow," International Journal of Non-Linear Mechanics, vol. 5, no. 4, pp. 659-685, 1970.

[19] R. C. Diprima and P. M. Eagles, “Amplification rates and torques for Taylor-vortex flows between rotating cylinders," Physics of Fluids, vol. 20, no. 2, pp. 171-175, 1977.

[20] P. R. Fenstermacher, H. L. Swinney, and J. P. Gollub, "Dynamical instabilities and the transition to the chaotic Taylor vortex flow," Journal of Fluid Mechanics, vol. 94, no. 1, pp. 103-128, 1979.

[21] K. Park, G. L. Crawford, and R. J. Donnelly, "Determination of transition in couette flow in finite geometries," Physical Review Letters, vol. 47, no. 20, pp. 1448-1450, 1981.

[22] K. Park and R. J. Donnelly, "Study of the transition to Taylor vortex flow," Physical Review A, vol. 24, no. 4, pp. 2277-2279, 1981.

[23] C. A. Jones, "On flow between counter-rotating cylinders," Journal of Fluid Mechanics, vol. 120, pp. 433-450, 1982.

[24] C. D. Andereck, R. Dickman, and H. L. Swinney, "New flows in a circular Couette system with corotating cylinders," Physics of Fluids, vol. 26, no. 6, pp. 1395-1401, 1983.

[25] P. S. Marcus, "Simulation of Taylor-Couette flow. Part 1. Numerical methods and comparison with experiment," Journal of Fluid Mechanics, vol. 146, pp. 45-64, 1984.

[26] C. D. Andereck, S. S. Liu, and H. L. Swinney, "Flow regimes in a circular Couette system with independently rotating cylinders," Journal of Fluid Mechanics, vol. 164, pp. 155-183, 1986.

[27] S. Chandrasekhar, "The stability of viscous flow between rotating cylinders in the presence of a magnetic field," Proceedings of the Royal Society. London. Series A, vol. 216, pp. 293-309, 1953.

[28] R. J. Donnelly and M. Ozima, "Hydromagnetic stability of flow between rotating cylinders," Physical Review Letters, vol. 4, no. 10, pp. 497-498, 1960.

[29] R. J. Donnelly and M. Ozima, "Experiments on the stability of flow between rotating cylinders in the presence of magnetic field," Proceedings of the Royal Society A, vol. 266, pp. 272-286, 1962.

[30] R. J. Donnelly and D. R. Caldwell, "Experiments on the stability of hydromagnetic Couette flow," Journal of Fluid Mechanics, vol. 19, pp. 257-263, 1964.

[31] A. Brahme, "On the hydromagnetic stability of a nonuniformly rotating fluid," Physica Scripta, vol. 2, pp. 108-112, 1970.

[32] H. Ji, J. Goodman, and A. Kageyama, "Magnetorotational instability in a rotating liquid metal annulus," Monthly Notices of the Royal Astronomical Society, vol. 325, no. 2, pp. L1-L5, 2001.

[33] J. Goodman and H. Ji, "Magnetorotational instability of dissipative Couette flow," Journal of Fluid Mechanics, vol. 462, pp. 365-382, 2002.

[34] A. P. Willis and C. F. Barenghi, "Hydromagnetic Taylor-Couette flow: numerical formulation and comparison with experiment," Journal of Fluid Mechanics, vol. 463, pp. 361-375, 2002.

[35] J. Szklarski and G. Rüdiger, "Nonlinear simulations of magnetic Taylor-Couette flow with current-free helical magnetic field," Astronomische Nachrichten, vol. 327, no. 9, pp. 844-849, 2006.

[36] J. Szklarski and G. Rüdiger, "Ekman-Hartmann layer in a magnetohydrodynamic Taylor-Couette flow," Physical Review E, vol. 76, no. 6, Article ID 066308, p. 8, 2007.

[37] J. D. Jackson, Classical Electrodynamics, John Wiley \& Sons, New York. NY, USA, 2nd edition, 1975.

[38] J. C. Leong, N. M. Brown, and F. C. Lai, "Mixed convection from an open cavity in a horizontal channel," International Communications in Heat and Mass Transfer, vol. 32, no. 5, pp. 583-592, 2005. 


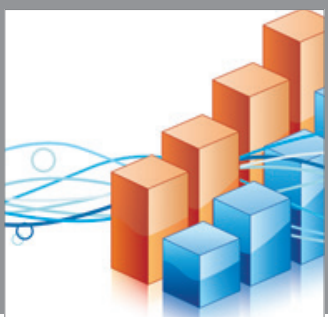

Advances in

Operations Research

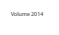

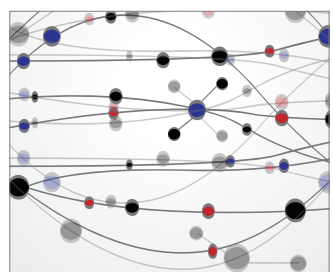

\section{The Scientific} World Journal
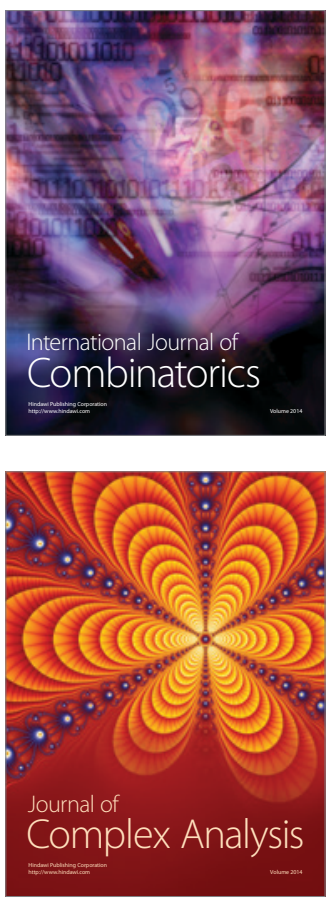

International Journal of

Mathematics and

Mathematical

Sciences
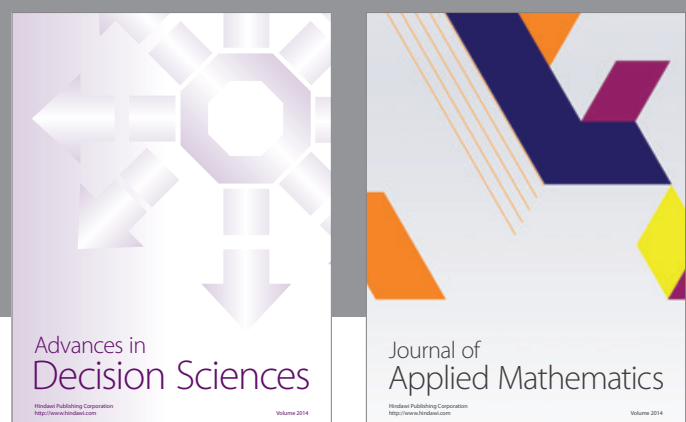

Journal of

Applied Mathematics
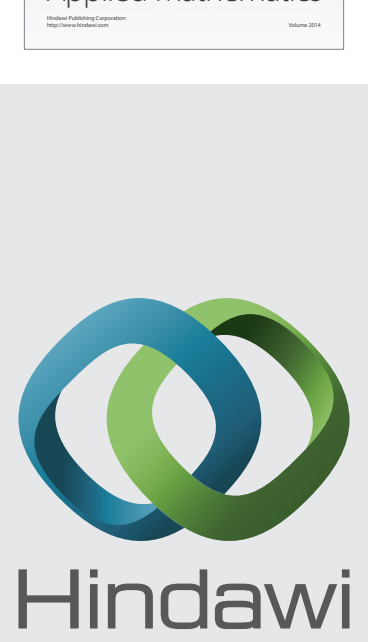

Submit your manuscripts at http://www.hindawi.com
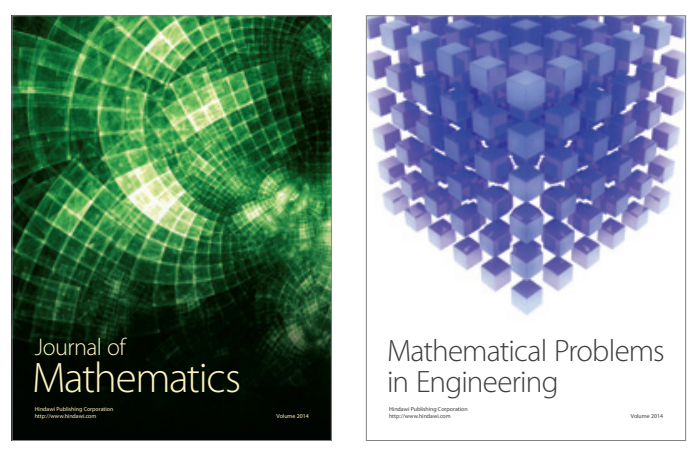

Mathematical Problems in Engineering
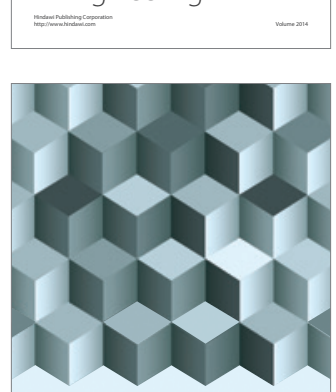

Journal of

Function Spaces
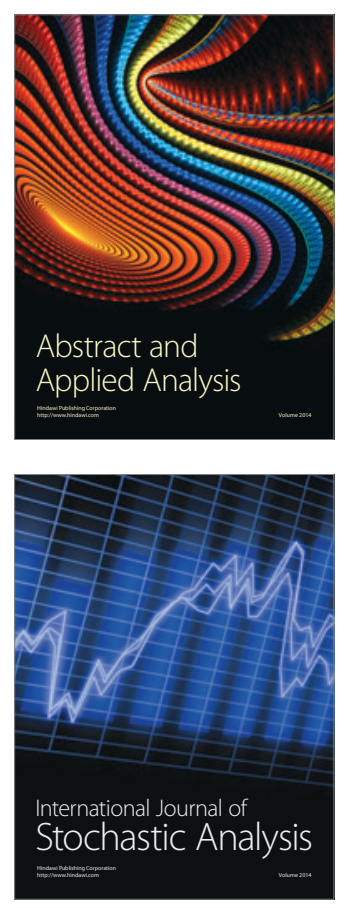

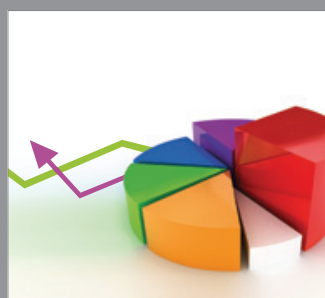

ournal of

Probability and Statistics

Promensencen
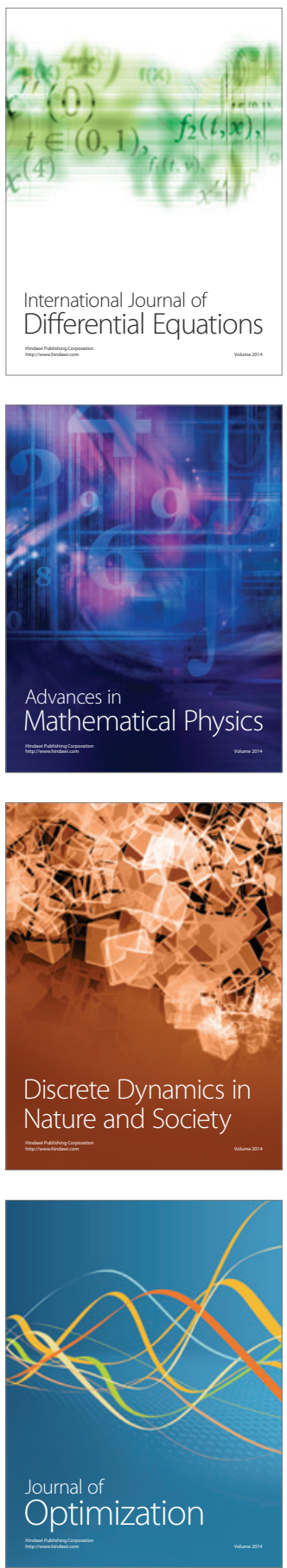